\title{
Implementing Telemedicine in Pediatric Primary Care During the COVID-19 Pandemic
}

Katherine E. Shedlock, MD - Benjamin N. Fogel, MD - Nicole M. Hackman, MD

The COVID-19 pandemic brought many important changes to modern medicine. While the world is adapting to new ways of life, medicine has been forced to deliver high-quality care while promoting and practicing social distancing. One aspect that has been transformed and propelled years into the future is the delivery of telemedicine. The American Academy of Pediatrics (AAP) provided a wealth of resources for health care providers who are new to using telemedicine.-4 However, one key question has gone unanswered: What pediatric primary care chief concerns are suitable for a telemedicine visit? The authors of this article have developed a list of pediatric chief concerns that effectively allow office personnel and patients to avoid unnecessary exposure to others and for patients to receive care in the comfort of their own home. The authors of this article also compiled a list of pediatric telehealth pearls, including creative ways to virtually obtain physical examination information.

\section{History}

Telehealth and telemedicine are now considered synonymous terms and are defined as "the use of medical information exchanged from one site to another via electronic communications to improve a patient's clinical health status." ${ }^{15}$ There are numerous benefits of telemedicine. Meeting virtually in alternative locations eliminates the need for reliable transportation and reduces the risk of acquiring an infectious illness by attending a visit in person. Telemedicine allows for remote care for patients in childcare facilities, schools, and juvenile detention facilities, and specialists can offer consultations from a distance. ${ }^{5}$ After proper implementation, it can also increase efficiency for the patient and the health care team. However, some barriers have slowed the adoption of telehealth, such as provider hesitancy, workflow disruption, technology expenses, and licensing and credentialing concerns..$^{5-7}$ Historically, one of the major limitations has been the lack of

\section{AFFILIATIONS:}

Department of Pediatrics, Penn State College of Medicine, Hershey, Pennsylvania

\section{CITATION:}

Shedlock KE, Fogel BN, Hackman NM. Implementing telemedicine in pediatric primary care during the COVID-19 pandemic. Consultant. Published online March 16, 2021. doi:10.25270/con.2021.03.00012

Received October 23, 2020. Accepted January 25, 2021.

\section{DISCLOSURES:}

The authors report no relevant financial relationships.

\section{ADDITIONAL CONTRIBUTIONS:}

We would like to recognize the important resources and guidance provided by the American Academy of Pediatrics (AAP) and the Pennsylvania chapter of the AAP, specifically Bonnie Offit, MD, who is the clinical advisor in digital health at the Children's Hospital of Philadelphia (CHOP) and a general pediatrician at the $\mathrm{CHOP}$ Care Network, who helped us develop our list of chief concerns that may be considered for telemedicine.

\section{CORRESPONDENCE:}

Katherine E. Shedlock, MD, Department of Pediatrics, Penn State College of Medicine, 500 University Drive, Hershey, PA 17033 (kshedlock@pennstatehealth.psu.edu)

Medicare reimbursement for telemedicine services except when providing care to an established beneficiary in a rural area. ${ }^{7}$ In addition, state Medicaid policies have been widely variable regarding regulations and reimbursement. ${ }^{7}$

When the novel coronavirus started to spread internationally, however, the potential impact on health care was obvious, and health care policies needed to quickly evolve. On March 17, 2020, in response to the urgent need for access to medical services while balancing the need for social distancing, the Centers for Medicare \& Medicaid Services announced that it had expanded opportunities for beneficiaries to receive Medicare telehealth services on a temporary and emergency basis. ${ }^{8}$ Under this monumental 1135 waiver, Medicare would reimburse for "office, hospital, and other visits furnished via telehealth across the country and including in patient's places of residence starting March 6, 2020." ${ }^{\prime 8}$ Policies in individual states started to follow suit and reduced barriers to reimbursement through Medicaid programs, the Children's Health Insurance Program, and private insurers. ${ }^{4}$

Previously, telehealth visits had to comply fully with the Health Insurance Portability and Accountability Act (HIPAA) of 1996. ${ }^{5}$ Private networks and electronic safeguards were necessary to ensure patients' health information and the telemedicine interaction were protected, but this is often logistically difficult. To speed the implementation of telemedicine during the COVID-19 pandemic, the Office for Civil Rights at the Department of Health and Human Services issued the Notification of Enforcement Discretion 
Table 1. Chief Concerns for Pediatric Primary Care Telehealth Visits

\section{ALLERGY (allergic conjunctivitis or rhinitis)}

\section{BEHAVIORAL HEALTH}

- Fussy baby/colic follow-up

- Medication check without suicidal/homicidal ideation or other condition requiring vital signs or examination

- Attention-deficit hyperactivity disorder

o Anxiety

- Depression

- Picky eater

- Problems in school

- Sleep concerns

BIRTH CONTROL INITIATION (had prior well-child check within 1 year)*

BREASTFEEDING/LACTATION

\section{DERMATOLOGY}

- Acne $e^{* *}$

- Minor cuts, burns, bites

- Rash/skin lesions (no fever, otherwise well)**

DYSURIA (afebrile and no abdominal pain)*

\section{FOLLOW-UP VISITS}

- Asthma

- Constipation

- Headache without vomiting or sensitivity to light or sound

- Hospital or urgent care follow-up

- Obesity

- Gastroesophageal reflux disease

\section{INFECTIOUS DISEASE}

- Congestion/rhinorrhea

- Cough without chest pain or shortness of breath

- Diarrhea without fever or blood in the stool

- Ear pain (during pandemic only)***

- Fever

- For fewer than 5 days

o Without chest pain, shortness of breath, dysuria, or concern for dehydration

- Pink eye or stye

- Sinus pressure

- Sore throat (during pandemic only)***

\section{WELL-CHILD CARE****}

* The patient should pick up a sterile urine cup from the office and deliver sample.

** Have a photo sent via electronic medical record prior to the visit, if possible.

*** Consider meeting the patient in the parking lot and performing examination/throat swab to confirm antibiotic need.

**** Ensure missed screenings (ie, vision and hearing) are tracked and completed after the pandemic. for Telehealth Remote Communications. ${ }^{9}$ This notice suddenly allowed the use of common video chat applications for virtual visits without the threat of penalties if the clinician is providing care in good faith.

\section{Implementation}

Revised billing policies, relaxation of HIPAA requirements, and the desire to promote social distancing for staff and patients, prompted health care teams across the country to rapidly implement telehealth services. Results of an April 2020 survey showed that nearly two-thirds of physicians had a $50 \%$ or more reduction in office visits during the pandemic, and nearly two-thirds of physicians started using telemedicine more than they did previously. ${ }^{10}$ According to results of another survey, $48 \%$ of physicians were treating patients via telemedicine compared with only $18 \%$ in $2018 .^{11}$ Patients have shown heightened interest in telemedicine as well. Teladoc, a large stand-alone telemedicine service, reported a $50 \%$ increase in service through March 20, 2020, compared with the week prior. $^{12}$ This abrupt shift to telemedicine left clinical teams searching for guidance. The AAP supported the expanded role, outlined processes for implementation, and provided resources that included a business plan., ${ }^{14}$

During implementation, one must consider health equity and the unique needs of the target patient population. Do the patients generally have access to the internet to participate in a video visit, or will access issues lead to health care discrimination? How will language translation services be utilized in order to ensure the service is inclusive? How will parents/guardians or patients with vision or hearing deficits be accommodated $?^{13}$ It is best to determine these processes in advance in order to avoid a delay in care for a patient who may need these special services.

Despite the wealth of implementation guidance now freely available, the question still remains: What types of visits can be done virtually? Visits 


\section{Table 2. Pearls for Pediatric Primary Care Telehealth Visits}

\section{VITAL SIGNS}

\section{WEIGHT}

- Weight can be obtained using a home scale.

- Adults can subtract their own weight after weighing themselves holding the child.

- Newborn scales are available for purchase for home use.

\section{HEIGHT/LENGTH}

- Height/length can be obtained using a measuring tape.

- One method: Lay the child on pavement, use chalk to mark each end, then measure the distance.

\section{HEAD CIRCUMFERENCE}

- Head circumference can be obtained using a piece of string.

\section{OTHER VITAL SIGNS}

- Temperature, heart rate, and respiratory rate can be obtained after simple instructions are given to the parent/guardian.

- Blood pressure can be obtained if a size-appropriate device is available at the home.

- Some smartwatches can report heart rate.

\section{SPECIAL SITUATIONS}

\section{CONFIDENTIAL DISCUSSIONS}

- Ask the patient to take the phone or video device to a private room.

\section{DERMATOLOGICAL CONCERNS}

- Request that photos are sent prior to the visit, especially for small lesions that are not seen well on video.

\section{FORMS}

- Send and receive screening and assessment forms electronically prior to the visit.

\section{THROAT CONCERNS}

- Request that photos be taken of the oropharynx while the patient is saying "ah."

that require history primarily, such as behavioral concerns or request for birth control initiation, and visits that require a minimal physical examination, such as for acne, are ideal for telemedicine. Many symptoms that could indicate an infectious condition can also be managed with telemedicine as long as certain exceptions are considered (Table 1). With a defined list, and under a physician's supervision, triage staff and schedulers can offer a telehealth visit as soon as a patient or caregiver calls about one of the chief concerns outlined in Table 1. This improves provider efficiency by reducing messages for provider approval to conduct visits virtually.

The current pandemic and need for social distancing must be weighed against the principal tenants of antibiotic stewardship and high-quality health care. Concerns of ear pain or sore throat may result in antibiotic prescriptions without physical examinations or confirmatory testing during the pandemic, but this practice would not be appropriate outside of this emergency situation. To more closely follow current antibiotic use guidelines while minimizing exposure, a health care provider could consider "drive-up" ear examinations and rapid strep testing. In fact, smartphone-enabled otoscopy applications exist to capture videos of the ear at home. Families may not be willing to pay for these devices, and their utility in the hands of untrained caregivers has been questioned. However, during this unique time, innovative ways to offer safe and effective care is desired. ${ }^{14-16}$ In the case of urogenital concerns, patients may be able to collect urine samples at home and deliver them to the laboratory, thereby reducing time spent in the facility. These processes may change as the crises abates, but many common chief concerns, such as constipation and behavioral health, will likely remain ideal visits to continue to offer virtually.

\section{Pearls to Delivering Pediatric Telemedicine}

Like any new practice, telemedicine becomes easier over time. As the process is being implemented, health care practitioners should consider the points and resourceful ways to obtain information from pediatric patients listed in Table 2.

\section{Conclusion}

During the COVID-19 pandemic and beyond, pediatric telemedicine will play an integral role in the delivery of health care. A variety of pediatric primary care chief concerns can successfully be evaluated via video platform with real-time communication. While balancing the need for social distancing and highquality care, health care providers will need to incorporate telemedicine as a component of practice for the foreseeable future. The routine use of telemedicine will improve health care practitioners' ability to provide flexible and efficient medical care to patients now and in the future.

\section{References}

1. Telehealth support: getting started in telehealth. American Academy of Pediatrics. Accessed April 17, 2020. https://www. aap.org/en-us/professional-resources/ 
practice-transformation/telehealth/Pages/ Getting-Started-in-Telehealth.aspx

2. Telehealth support: what is telehealth? American Academy of Pediatrics. Accessed April 17, 2020. https://www.aap.org/en-us/professional-resources/practice-transformation/ telehealth/Pages/compendium.aspx

3. COVID-19 pediatric provider resources \& information. American Academy of Pediatrics, Pennsylvania Chapter. Updated February 19, 2021. Accessed April 17, 2020. https://www. paaap.org/covid-19.html

4. Guidance on providing pediatric ambulatory services via telehealth during COVID-19. American Academy of Pediatrics. Updated January 6, 2021. Accessed April 15, 2020. https://services.aap.org/en/ pages/2019-novel-coronavirus-covid-19-infections/guidance-on-providing-pediatric-ambulatory-services-via-telehealth-during-covid-19/

5. Burke Jr BL, Hall RW; Section on Telehealth Care. Telemedicine: pediatric applications. Pediatrics. 2015;136(1):e293-e308. https:// doi.org/10.1542/peds.2015-1517

6. Brooks E, Turvey C, Augusterfer EF. Provider barriers to telemental health: obstacles overcome, obstacles remaining. Telemed J E Health. 2013;19(6):433-437. https://doi. org/10.1089/tmj.2013.0068

7. Telehealth policy barriers. Public Health Institute Center for Connected Health Policy. Published February 2018. Accessed April 17, 2020. https://www.telehealthpolicy.us/sites/ default/files/2018-09/TELEHEALTH\%20 POLICY\%20BARRIERS\%202018\%20 FINAL\%20.pdf

8. Medicare telemedicine health care provider fact sheet. US Centers for Medicare \& Medicaid Services. Published March 17, 2020. Accessed March 17, 2020. https://www.cms. gov/newsroom/fact-sheets/medicare-telemedicine-health-care-provider-fact-sheet

9. Notification of enforcement discretion for telehealth remote communications during the COVID-19 nationwide public health emergency. US Department of Health and Human Services. Reviewed January 20, 2021. Accessed March 31, 2020. https:// www.hhs.gov/hipaa/for-professionals/special-topics/emergency-preparedness/notification-enforcement-discretion-telehealth/ index.html\#

10. Murphy J. Doctors on edge as practice revenues plunge, survey finds. MDLinx. Published April 17, 2020. https://www. mdlinx.com/internal-medicine/article/6867

11. Survey: physician practice patterns changing as a result of COVID-19. News Release. Merritt Hawkins. April 22, 2020. Accessed May 5, 2020. https://www.merritthawkins. com/news-and-insights/media-room/ press/-Physician-Practice-Patterns-Changing-as-a-Result-of-COVID-19/

12. Olson P. Telemedicine, once a hard sell, can't keep up with demand. Wall St J. Published April 1, 2020. https://www.wsj.com/ articles/telemedicine-once-a-hard-sellcant-keep-up-with-demand-11585734425

13. HHS nondiscrimination notice. US Department of Health and Human Services. Reviewed July 18, 2020. Accessed May 5, 2020. https://www.hhs.gov/civil-rights/ for-individuals/nondiscrimination/index.html

14. Erkkola-Anttinen N, Tähtinen PA, Laine MK, Ruohola A. Parental role in the diagnostics of otitis media: can parents be taught to use tympanometry reliably? Int J Pediatr Otorhinolaryngol. 2014;78(7):1036-1039. https:// doi.org/10.1016/j.ijporl.2014.03.035

15. Erkkola-Anttinen $\mathrm{N}$, Irjala $\mathrm{H}$, Laine $\mathrm{MK}$, Tähtinen PA, Löyttyniemi E, Ruohola A. Smartphone otoscopy performed by parents. Telemed J E Health. 2019;25(6):477-484. https://doi.org/10.1089/tmj.2018.0062

16. Shah MU, Sohal M, Valdez TA, Grindle CR. iPhone otoscopes: currently available, but reliable for tele-otoscopy in the hands of parents? Int J Pediatr Otorhinolaryngol. 2018;106:59-63. https://doi.org/10.1016/j. ijporl.2018.01.003 